\title{
New Methods of Finite-Time Synchronization for a Class of Fractional-Order Delayed Neural Networks
}

\author{
Weiwei Zhang, ${ }^{1}$ Jinde Cao, ${ }^{2,3}$ Ahmed Alsaedi, ${ }^{4}$ and Fuad E. Alsaadi ${ }^{5}$ \\ ${ }^{1}$ School of Mathematics and Computational Science, Anqing Normal University, Anqing 246011, China \\ ${ }^{2}$ School of Mathematics, Southeast University, Nanjing 210096, China \\ ${ }^{3}$ School of Mathematics and Statistics, Shandong Normal University, Jinan 250014, China \\ ${ }^{4}$ Department of Mathematics, King Abdulaziz University, Jeddah 21589, Saudi Arabia \\ ${ }^{5}$ Department of Electrical and Computer Engineering, King Abdulaziz University, Jeddah 21589, Saudi Arabia
}

Correspondence should be addressed to Weiwei Zhang; wwzhahu@aliyun.com

Received 22 March 2017; Accepted 10 May 2017; Published 6 June 2017

Academic Editor: R. Aguilar-López

Copyright (c) 2017 Weiwei Zhang et al. This is an open access article distributed under the Creative Commons Attribution License, which permits unrestricted use, distribution, and reproduction in any medium, provided the original work is properly cited.

Finite-time synchronization for a class of fractional-order delayed neural networks with fractional order $\alpha, 0<\alpha \leq 1 / 2$ and $1 / 2<\alpha<1$, is investigated in this paper. Through the use of Hölder inequality, generalized Bernoulli inequality, and inequality skills, two sufficient conditions are considered to ensure synchronization of fractional-order delayed neural networks in a finitetime interval. Numerical example is given to verify the feasibility of the theoretical results.

\section{Introduction}

Since the 17th century, the theory of fractional calculus was mainly focused on the pure theoretical field of mathematics $[1,2]$. In the past two decades, it has been found that the dynamical behaviors of many systems can be described by the fractional calculus. Furthermore, fractional-order models can help exhibit the dynamical behaviors of systems. In fact, many physical systems show fractional dynamical behaviors because of special properties [3-11].

Fractional-order neural networks have attracted great attention due to their potential properties of memory and hereditary. Particularly, the dynamical analysis of fractionalorder neural networks can be used to describe the dynamical characteristics of neural networks. For instance, synchronization is considered as an important topic, as reported in $[12,13]$, where chaotic synchronization of fractional-order neural networks was proposed. Mittag-Leffler stability and synchronization of memristor-based fractional-order neural networks were discussed in [14]. In addition, the results for stability analysis and synchronization of fractional-order networks were presented in [15-23].

However, the majority of the results were demonstrated to ensure the asymptotic stability of error systems.
Asymptotic synchronization indicates that the trajectories of the slave system reach to the trajectories of the master system over the infinite horizon. In fact, it is more desirable that the networks can reach synchronization in a finite-time in physical and engineering systems, achieving an optimality in convergence time. Thus, it is necessary to study the finite-time synchronization of neural networks. In order to achieve faster synchronization in control systems, an effective finite-time control method is utilized. Some important results on finitetime synchronization were demonstrated on integer-order systems [24-28]. Note that time delay [29-31] occurs in many physical and engineering systems. So it is natural to study fractional-order systems with delays. Till now few results are obtained with the consideration of the finite-time stability and synchronization of fractional-order neural networks with time delays [32, 33]. For instance, finite-time synchronization of fractional-order memristor-based neural networks with time delays was considered by using Laplace transform, such as the generalized Gronwall inequality and Mittag-Leffler functions, in [33].

Motivated by the above discussion, the main goal of this paper is to adopt new methods and obtain some new sufficient conditions that can assist master-slave systems to 
achieve the finite-time synchronization of fractional delayed neural networks with orders $0<\alpha \leq 1 / 2$ and $1 / 2<\alpha<1$.

Throughout the paper, denote $\|x\|=\sum_{i=1}^{n}\left|x_{i}\right|(i=$ $1, \ldots, n)$ and $\|A\|=\max _{j} \sum_{i=1}^{n}\left|a_{i j}\right|(i, j=1, \ldots, n)$ which are the Euclidean vector norm and the matrix norm, respectively; $x_{i}$ and $a_{i j}$ are the element of the vector $x$ and the matrix $A$.

\section{Preliminaries and Model Description}

There are some definitions of the fractional-order integrals and derivatives. Due to the advantages of the Caputo fractional derivative, the definition of Caputo derivative is used in this paper.

Definition 1 (see [1]). The fractional integral with noninteger order $\alpha>0$ of a function $x(t)$ is defined by

$$
D^{-\alpha} x(t)=\frac{1}{\Gamma(\alpha)} \int_{0}^{t}(t-\tau)^{\alpha-1} x(\tau) d \tau,
$$

where $t \geq 0, \Gamma(\cdot)$ is the Gamma function, and $\Gamma(s)=$ $\int_{0}^{\infty} t^{s-1} e^{-t} d t$

Definition 2 (see [1]). The Caputo derivative of fractional order $\alpha$ of a function $x(t)$ is defined by

$$
D^{\alpha} x(t)=\frac{1}{\Gamma(n-\alpha)} \int_{0}^{t}(t-\tau)^{n-\alpha-1} x^{(n)}(\tau) d \tau,
$$

where $t \geq 0 ; n-1<\alpha<n \in Z^{+}$.

In this paper, we consider a class of fractional-order neural networks with time delay as master system, which is described by

$$
\begin{aligned}
D^{\alpha} x_{i}(t)= & -c_{i} x_{i}(t)+\sum_{j=1}^{n} a_{i j}(t) f_{j}\left(x_{j}(t)\right) \\
& +\sum_{j=1}^{n} b_{i j}(t) g_{j}\left(x_{j}(t-\tau)\right)+I_{i},
\end{aligned}
$$

or equivalently

$$
\begin{aligned}
D^{\alpha} x(t)= & -C x(t)+A(t) f(x(t))+B(t) g(x(t-\tau)) \\
& +I
\end{aligned}
$$

for $t \in J=[0, T](T>0)$, where $0<\alpha<1, i=1,2, \ldots, n, n$ is the number of units in a neural network, $x_{i}(t)$ corresponds to the state of the $i$ th unit at time $t$ and denotes $x(t)=$ $\left(x_{1}(t), \ldots, x_{n}(t)\right)^{T} \in R^{n}$, and $C=\operatorname{diag}\left(c_{i}>0\right)$ is the selfregulating parameter of the neurons. $I=\left(I_{1}, I_{2}, \ldots, I_{n}\right)^{T}$ represents the external input; $A(t)=\left(a_{i j}(t)\right)_{n \times n}$ and $B(t)=$ $\left(b_{i j}(t)\right)_{n \times n}$ are the connective weights matrix in the presence and absence of delay, respectively. Functions $f_{j}\left(x_{j}(t)\right)$ and $g_{j}\left(x_{j}(t)\right)$ denote the output of the $j$ th unit at time $t$ and $t-\tau$, respectively, where $\tau>0$ is the transmission delay and denotes $f(x(t))=\left(f_{1}\left(x_{1}(t)\right), \ldots, f_{n}\left(x_{n}(t)\right)\right)^{T}, g(x(t))=$ $\left(g_{1}\left(x_{1}(t)\right), \ldots, g_{n}\left(x_{n}(t)\right)\right)^{T}$.

The initial conditions associated with system (1) are of the form $x_{i}(t)=\psi_{i}(t), t \in[-\tau, 0](i=1, \ldots, n)$, where $\psi_{i}(t)$ denotes the real-valued continuous function defined on $[-\tau, 0]$, with the norm given by $\|\psi\|=\sup _{s \in[-\tau, 0]}\|\psi(s)\|$.

The slave system is given:

$$
\begin{aligned}
D^{\alpha} y_{i}(t)= & -c_{i} y_{i}(t)+\sum_{j=1}^{n} a_{i j}(t) f_{j}\left(y_{j}(t)\right) \\
& +\sum_{j=1}^{n} b_{i j}(t) g_{j}\left(y_{j}(t-\tau)\right)-u_{i}(t)+I_{i},
\end{aligned}
$$

or equivalently

$$
\begin{aligned}
D^{\alpha} y(t)= & -C y(t)+A(t) f(y(t))+B(t) g(y(t-\tau)) \\
& -U(t)+I
\end{aligned}
$$

where $y(t)=\left(y_{1}(t), \ldots, y_{n}(t)\right)^{T} \in R^{n}$ is the state vector of the system response and $U(t)=\left(u_{1}(t), \ldots, u_{n}(t)\right)^{T}$ is a suitable controller. The initial conditions associated with system (3) are of the form $y_{i}(t)=\pi_{i}(t), t \in[-\tau, 0](i=1, \ldots, n)$, where $\pi_{i}(t)$ denotes the real-valued continuous function defined on $[-\tau, 0]$, with the norm given by $\|\pi\|=\sup _{s \in[-\tau, 0]}\|\pi(s)\|$.

For generalities, the following definition, assumptions, and lemmas are presented.

Definition 3. System (1) is said to be synchronized with system (3) in a finite-time with respect to $\{0, \delta, \varepsilon, T\}$, for a suitable designed controller $u_{i}(t)$, if and only if $\|\pi(0)-\psi(0)\| \leq$ $\delta$, implying $\left\|e_{i}(t)\right\|=\left\|y_{i}(t)-x_{i}(t)\right\|<\varepsilon, \forall t \in[0, T]$, where $\delta, \epsilon, T$ are real positive numbers and $\delta<\varepsilon$.

Assumption 4. The neuron activation functions $f(x), g(x)$ are Lipschitz continuous, with the existence of positive constants $H, K$, such that

$$
\begin{gathered}
\|f(u)-f(v)\| \leq H\|u-v\|, \\
\|g(u)-g(v)\| \leq K\|u-v\|,
\end{gathered}
$$

for all $u, v \in R^{n}$.

Assumption 5. $a_{i j}(t)$ and $b_{i j}(t)$ are continuous and bounded functions defined on $R^{+}$, and let $A=\sup _{t \geq 0}\|A(t)\|, B=$ $\sup _{t \geq 0}\|B(t)\|$.

Lemma 6 (Hölder inequality [34]). Assume that $p, q>1$ and $1 / p+1 / q=1$, and if $|f(\cdot)|^{p},|g(\cdot)|^{q} \in L^{1}(E)$, then $f(\cdot) g(\cdot) \in$ $L^{1}(E)$ and

$$
\begin{aligned}
& \int_{E}|f(x) g(x)| d x \\
& \quad \leq\left(\int_{E}|f(x)|^{p} d x\right)^{1 / p}\left(\int_{E}|g(x)|^{q} d x\right)^{1 / q}
\end{aligned}
$$

where $L^{1}(E)$ is the Banach space of all Lebesgue measurable functions $f: E \rightarrow R$ with $\int_{E}|f(x)| d x<\infty$. 
Let $p=q=2$; it reduces to the Cauchy-Schwartz inequality as follows:

$$
\begin{aligned}
& \left(\int_{E}|f(x) g(x)| d x\right)^{2} \\
& \quad \leq\left(\int_{E}|f(x)|^{2} d x\right)\left(\int_{E}|g(x)|^{2} d x\right) .
\end{aligned}
$$

Lemma 7 (generalized Bernoulli inequality [34]). If $k \in R^{+}$, $x<1$ and $x \neq 0$, then, for $0<k<1,(1-x)^{k}<1-k x$, or $\left(1-(1-x)^{k}\right)^{-1}<(k x)^{-1}$.

Lemma 8 (see [35]). If $x(t) \in C^{m}[0, \infty)$ and $m-1<\alpha<m \in$ $z^{+}$, then

(1) $D^{-\alpha} D^{-\beta} x(t)=D^{-(\alpha+\beta)} x(t), \alpha, \beta \geq 0$,

(2) $D^{\alpha} D^{-\beta} x(t)=x(t), \alpha=\beta \geq 0$,

(3) $D^{-\alpha} D^{\beta} x(t)=x(t)-\sum_{k=0}^{m-1}\left(t^{k} / k !\right) x^{(k)}(0), \alpha=\beta \geq 0$.

Lemma 9 (see [36]). Let $u(t), \omega(t), v(t)$, and $h(t)$ be nonnegative continuous functions on $R^{+}$and let $r \geq 1$ be a real number. If

$$
u(t) \leq u_{0}(t)+\omega(t)\left(\int_{0}^{t} v(s) u^{r}(s) d s\right)^{1 / r}, \quad t \in R^{+}
$$

then

$$
\begin{aligned}
& \int_{0}^{t} v(s) u^{r}(s) d s \\
& \quad \leq\left[1-(1-W(t))^{1 / r}\right]^{-r} \int_{0}^{t} v(s) u_{0}^{r}(s) W(s) d s,
\end{aligned}
$$

where $W(t)=\exp \left(-\int_{0}^{t} v(s) \omega^{r}(s) d s\right)$.

\section{Finite-Time Synchronization}

In this section, master-slave finite-time synchronization of delayed fractional-order neural networks is discussed. The aim here is to design a suitable controller that can achieve the synchronization between the slave system and the master system.

Let $e_{i}(t)=y_{i}(t)-x_{i}(t)(i=1,2, \ldots, n)$ be the synchronization errors.

Select the linear control input functions $u_{i}(t)$ as the following form:

$$
u_{i}(t)=\eta_{i}\left(y_{i}(t)-x_{i}(t)\right)
$$

where each $\eta_{i}>0(i=1, \ldots, n)$ denotes the control gain.

Then the error systems are obtained:

$$
\begin{aligned}
D^{\alpha} e_{i}(t) & \\
= & -\left(c_{i}+\eta_{i}\right) e_{i}(t) \\
& +\sum_{j=1}^{n} a_{i j}(t)\left[f_{j}\left(y_{j}(t)\right)-f_{j}\left(x_{j}(t)\right)\right] \\
& +\sum_{j=1}^{n} b_{i j}(t)\left[g_{j}\left(y_{j}(t-\tau)\right)-g_{j}\left(x_{j}(t-\tau)\right)\right] .
\end{aligned}
$$

The vector form is as follows:

$$
\begin{aligned}
D^{\alpha} e(t)= & -\Omega e(t)+A(t)[f(y(t))-f(x(t))] \\
& +B(t)[g(y(t-\tau))-g(x(t-\tau))],
\end{aligned}
$$

where $e(t)=\left(e_{1}(t), \ldots, e_{n}(t)\right)^{T}$ and $\Omega=\operatorname{diag}\left(c_{1}+\eta_{1}, \ldots, c_{n}+\right.$ $\left.\eta_{n}\right)$.

The initial conditions $e(t)$ of system (14) are of the following form:

$$
\begin{aligned}
e_{i}(t)=\pi_{i}(t)-\psi_{i}(t)=\phi_{i}(t), & \\
& t \in[-\tau, 0](i=1, \ldots, n) .
\end{aligned}
$$

Theorem 10. When $1 / 2<\alpha<1$, suppose that Assumptions 4 and 5 hold, if

$$
\left[1+N e^{t}+2(1+N) e^{\left[\left(M+N e^{-\tau}\right)^{2}+1\right] t}\right]<\frac{\varepsilon}{\delta},
$$

$$
t \in[0, T],
$$

where $M=(\|\Omega\|+A H) \sqrt{2 \Gamma(2 \alpha-1)} / \Gamma(\alpha) 2^{\alpha}, N=$ $B K \sqrt{2 \Gamma(2 \alpha-1)} / \Gamma(\alpha) 2^{\alpha}$; master system (3) is synchronized with slave system (5) in a finite-time with respect to $\{0, \delta, \varepsilon, T\}$ under the control (12).

Proof. Let $e_{0}=\phi(0)$ be the initial condition of system (14), based on Lemma 8, the solution of system (7) in the form of the equivalent Volterra fractional integral equation is as follows:

$$
\begin{aligned}
& e(t)=e_{0}+D^{-\alpha}[-\Omega e(t)+A(t)(f(y(t))-f(x(t))) \\
& +B(t)(g(y(t-\tau))-g(x(t-\tau)))]=e_{0}+\frac{1}{\Gamma(\alpha)} \\
& \quad \cdot \int_{0}^{t}(t-s)^{\alpha-1}[-\Omega e(s) \\
& \quad+A(s)(f(y(s))-f(x(s))) \\
& +B(s)(g(y(s-\tau))-g(x(s-\tau)))] d s .
\end{aligned}
$$

Taking the norm $\|\cdot\|$ on both sides of the above system, according to the Assumptions 4 and 5, gives the following:

$$
\begin{aligned}
& \|e(t)\| \leq\left\|e_{0}\right\|+\frac{1}{\Gamma(\alpha)} \int_{0}^{t}(t-s)^{\alpha-1}[\|-\Omega e(s) \\
& +A(s)(f(y(s))-f(x(s))) \\
& +B(s)(g(y(s-\tau))-g(x(s-\tau))) \|] d s \\
& \leq\|\phi(0)\|+\frac{1}{\Gamma(\alpha)} \int_{0}^{t}(t-s)^{\alpha-1}[\|\Omega\|\|e(s)\| \\
& +A H\|e(s)\|+B K\|e(s-\tau)\|] d s=\|\phi(0)\| \\
& +\frac{(\|\Omega\|+A H)}{\Gamma(\alpha)} \int_{0}^{t}(t-s)^{\alpha-1} e^{s} e^{-s}\|e(s)\| d s+\frac{B K}{\Gamma(\alpha)} \\
& . \int_{0}^{t}(t-s)^{\alpha-1} e^{s} e^{-s}\|e(s-\tau)\| d s .
\end{aligned}
$$


By using the Cauchy-Schwartz inequality, (18) gets the following:

$$
\begin{aligned}
& \|e(t)\| \leq\|\phi(0)\| \\
& +\frac{(\|\Omega\|+A H)}{\Gamma(\alpha)}\left(\int_{0}^{t}(t-s)^{2(\alpha-1)} e^{2 s} d s\right)^{1 / 2} \\
& \cdot\left(\int_{0}^{t} e^{-2 s}\|e(s)\|^{2} d s\right)^{1 / 2} \\
& +\frac{B K}{\Gamma(\alpha)}\left(\int_{0}^{t}(t-s)^{2(\alpha-1)} e^{2 s} d s\right)^{1 / 2} \\
& \cdot\left(\int_{0}^{t} e^{-2 s}\|e(s-\tau)\|^{2}\right)^{1 / 2} d s=\|\phi(0)\| \\
& +\left(\int_{0}^{t}(t-s)^{2(\alpha-1)} e^{2 s} d s\right)^{1 / 2} \\
& +\left[\frac{(\|\Omega\|+A H)}{\Gamma(\alpha)}\left(\int_{0}^{t} e^{-2 s}\|e(s)\|^{2} d s\right)^{1 / 2} d s\right. \\
& \left.+\frac{B K}{\Gamma(\alpha)}\left(\int_{0}^{t} e^{-2 s}\|e(s-\tau)\|^{2}\right)^{1 / 2} d s\right] .
\end{aligned}
$$

Note that

$$
\begin{aligned}
\int_{0}^{t}(t-s)^{2(\alpha-1)} e^{2 s} d s & =\int_{0}^{t} z^{(2 \alpha-2)} e^{2(t-z)} d z \\
& =e^{2 t} \int_{0}^{t} z^{(2 \alpha-2)} e^{-2 z} d z \\
& =\frac{2 e^{2 t}}{4^{\alpha}} \int_{0}^{2 t} \theta^{(2 \alpha-2)} e^{-\theta} d \theta \\
& <\frac{2 e^{2 t}}{4^{\alpha}} \Gamma(2 \alpha-1) .
\end{aligned}
$$

Noting that $e(t)=\phi(t)(t \in[-\tau, 0])$ and $\|\phi\|=$ $\sup _{\theta \in[-\tau, 0]}\|\phi(\theta)\|$, one obtains

$$
\begin{aligned}
& \int_{0}^{t} e^{-2 s}\|e(s-\tau)\|^{2} d s \leq e^{-2 \tau} \int_{-\tau}^{t} e^{-2 u}\|e(u)\|^{2} d u \\
& \quad=e^{-2 \tau}\left[\int_{-\tau}^{0} e^{-2 u}\|e(u)\|^{2} d u+\int_{0}^{t} e^{-2 u}\|e(u)\|^{2} d u\right] \\
& \quad \leq\|\phi\|^{2}+e^{-2 \tau} \int_{0}^{t} e^{-2 s}\|e(s)\|^{2} d s .
\end{aligned}
$$

Then

$\|e(t)\| \leq\|\phi(0)\|$

$$
+\frac{e^{t} \sqrt{2 \Gamma(2 \alpha-1)}}{2^{\alpha}}\left[\frac{(\|\Omega\|+A H)}{\Gamma(\alpha)}\left(\int_{0}^{t} e^{-2 s}\|e(s)\|^{2} d s\right)^{1 / 2}\right.
$$

$$
\begin{aligned}
& \left.+\frac{B K}{\Gamma(\alpha)}\left(\|\phi\|^{2}+e^{-2 \tau} \int_{0}^{t} e^{-2 s}\|e(s)\|^{2}\right)^{1 / 2} d s\right]<(\|\phi\| \\
& \left.+\frac{e^{t} \sqrt{2 \Gamma(2 \alpha-1)} B K}{2^{\alpha} \Gamma(\alpha)}\|\phi\|\right) \\
& +e^{t}\left[\frac{\sqrt{2 \Gamma(2 \alpha-1)}(\|\Omega\|+A H)}{2^{\alpha} \Gamma(\alpha)}\right. \\
& \left.+\frac{\sqrt{2 \Gamma(2 \alpha-1)} B K}{2^{\alpha} \Gamma(\alpha)} e^{-\tau}\right]\left(\int_{0}^{t} e^{-2 s}\|e(s)\|^{2} d s\right)^{1 / 2} d s .
\end{aligned}
$$

Let $M=\sqrt{2 \Gamma(2 \alpha-1)}(\|\Omega\|+A H) / 2^{\alpha} \Gamma(\alpha)$ and $N=$ $\sqrt{2 \Gamma(2 \alpha-1)} B K / 2^{\alpha} \Gamma(\alpha)$.

Then

$$
\begin{aligned}
& \|e(t)\| e^{-t} \\
& \leq\left(\|\phi\| e^{-t}+N\|\phi\|\right) \\
& \quad+\left(M+N e^{-\tau}\right)\left(\int_{0}^{t} e^{-2 s}\|e(s)\|^{2} d s\right)^{1 / 2} d s .
\end{aligned}
$$

According to Lemmas 7 and 9, one has

$$
\begin{gathered}
\|e(t)\| e^{-t} \leq\left(\|\phi\| e^{-t}+N\|\phi\|\right)+\left(M+N e^{-\tau}\right) \\
\cdot\left[\left(1-\left(1-e^{-\left(M+N e^{-\tau}\right)^{2} t}\right)^{1 / 2}\right)^{-2}\right. \\
\left.\cdot \int_{0}^{t}\left(\|\phi\| e^{-s}+N\|\phi\|\right)^{2} e^{-\left(M+N e^{-\tau}\right)^{2} s} d s\right]^{1 / 2} \\
\leq\left(\|\phi\| e^{-t}+N\|\phi\|\right)+\left(M+N e^{-\tau}\right) 2 e^{\left(M+N e^{-\tau}\right)^{2} t} \\
\cdot\left[\int_{0}^{t}\left(\|\phi\| e^{-s}+N\|\phi\|\right)^{2} e^{-\left(M+N e^{-\tau}\right)^{2} s} d s\right]^{1 / 2} \\
\leq\left(\|\phi\| e^{-t}+N\|\phi\|\right)+2(1+N)\|\phi\| e^{\left(M+N e^{-\tau}\right)^{2} t} \\
=\left[e^{-t}+N+2(1+N) e^{\left(M+N e^{-\tau}\right)^{2} t}\right]\|\phi\| .
\end{gathered}
$$

Therefore

$$
\|e(t)\| \leq\left[1+N e^{t}+2(1+N) e^{\left[\left(M+N e^{-\tau}\right)^{2}+1\right] t}\right]\|\phi\| .
$$

Hence, if (16) is satisfied and $\|\phi\|<\delta$, then $\|e(t)\|<\varepsilon$; master system (3) is synchronized with slave system (5) in a finitetime.

Theorem 11. When $0<\alpha \leq 1 / 2$, suppose that Assumptions 4 and 5 hold, if

$$
1+\bar{N} e^{t}+q(1+\bar{N}) e^{\left[\left(\bar{M}+\bar{N} e^{-\tau}\right)^{q}+1\right] t}<\frac{\varepsilon}{\delta}, \quad t \in[0, T],
$$


where $\bar{M}=(\|\Omega\|+A H)\left[\Gamma\left(\alpha^{2}\right) / \Gamma^{p}(\alpha) p^{\alpha^{2}}\right]^{1 / p}, \bar{N}=$ $B K\left[\Gamma\left(\alpha^{2}\right) / \Gamma^{p}(\alpha) p^{\alpha^{2}}\right]^{1 / p}$ and $p=1+\alpha, q=1+1 / \alpha$; then master system (3) is synchronized with slave system (5) in a finite-time with respect to $\{0, \delta, \varepsilon, T\}$ under control (12).

Proof. Similar to Theorem 10, we can obtain the following estimation:

\section{$\|e(t)\| \leq\|\phi(0)\|$}

$$
\begin{aligned}
& +\frac{(\|\Omega\|+A H)}{\Gamma(\alpha)} \int_{0}^{t}(t-s)^{\alpha-1} e^{s} e^{-s}\|e(s)\| d s \\
& +\frac{B K}{\Gamma(\alpha)} \int_{0}^{t}(t-s)^{\alpha-1} e^{s} e^{-s}\|e(s-\tau)\| d s .
\end{aligned}
$$

Let $p=1+\alpha, q=1+1 / \alpha$; obviously, $p, q>1$ and $1 / p+1 / q=1$, and following the Hölder inequality, we get

$$
\begin{aligned}
& \|e(t)\| \leq\|\phi(0)\| \\
& +\frac{(\|\Omega\|+A H)}{\Gamma(\alpha)}\left[\int_{0}^{t}(t-s)^{p(\alpha-1)} e^{p s} d s\right]^{1 / p} \\
& \cdot\left[\int_{0}^{t} e^{-q s}\|e(s)\|^{q} d s\right]^{1 / q} \\
& +\frac{B K}{\Gamma(\alpha)}\left[\int_{0}^{t}(t-s)^{p(\alpha-1)} e^{p s} d s\right]^{1 / p} \\
& .\left[\int_{0}^{t} e^{-q s}\|e(s-\tau)\|^{q} d s\right]^{1 / q}=\|\phi(0)\| \\
& +\left[\int_{0}^{t}(t-s)^{p(\alpha-1)} e^{p s} d s\right]^{1 / p} \\
& .\left[\frac{(\|\Omega\|+A H)}{\Gamma(\alpha)}\left(\int_{0}^{t} e^{-q s}\|e(s)\|^{q} d s\right)^{1 / q}\right. \\
& \left.+\frac{B K}{\Gamma(\alpha)}\left(\int_{0}^{t} e^{-q s}\|e(s-\tau)\|^{q} d s\right)^{1 / q}\right] .
\end{aligned}
$$

Since

$$
\begin{aligned}
& \int_{0}^{t}(t-s)^{p(\alpha-1)} e^{p s} d s=e^{p t} \int_{0}^{t} z^{p(\alpha-1)} e^{-p z} d z \\
& =\frac{e^{p t}}{p^{p(\alpha-1)+1}} \int_{0}^{p t} u^{p(\alpha-1)} e^{-u} d u \leq \frac{e^{p t}}{p^{\alpha^{2}}} \Gamma\left(\alpha^{2}\right), \\
& \int_{0}^{t} e^{-q s}\|e(s-\tau)\|^{q} d s \leq e^{-q \tau} \int_{-\tau}^{t} e^{-q u}\|e(u)\|^{q} d u
\end{aligned}
$$

$$
\begin{aligned}
& =e^{-q \tau}\left[\int_{-\tau}^{0} e^{-q u}\|e(u)\|^{q} d u+\int_{0}^{t} e^{-q u}\|e(u)\|^{q} d u\right] \\
& \leq\|\phi\|^{q}+e^{-q \tau} \int_{0}^{t} e^{-q s}\|e(s)\|^{q} d s .
\end{aligned}
$$

then

$$
\begin{aligned}
& \|e(t)\| \leq\|\phi(0)\|+\left[\frac{\Gamma\left(\alpha^{2}\right)}{p^{\alpha^{2}}}\right]^{1 / p} \\
& \cdot e^{t}\left[\frac{(\|\Omega\|+\mathrm{AH})}{\Gamma(\alpha)}\left(\int_{0}^{t} e^{-q s}\|e(s)\|^{q} d s\right)^{1 / q}\right. \\
& \left.+\frac{B K}{\Gamma(\alpha)}\left(\|\phi\|^{q}+e^{-q \tau} \int_{0}^{t} e^{-q s}\|e(s)\|^{q} d s\right)^{1 / q}\right] \\
& \leq\left[\|\phi\|+B K\left(\frac{\Gamma\left(\alpha^{2}\right)}{\Gamma^{p}(\alpha) p^{\alpha^{2}}}\right)^{1 / p} e^{t}\|\phi\|\right] \\
& +e^{t}\left[(\|\Omega\|+A H)\left(\frac{\Gamma\left(\alpha^{2}\right)}{\Gamma^{p}(\alpha) p^{\alpha^{2}}}\right)^{1 / p}\right. \\
& \left.+B K\left(\frac{\Gamma\left(\alpha^{2}\right)}{\Gamma^{p}(\alpha) p^{\alpha^{2}}}\right)^{1 / p} e^{-\tau}\right] \\
& .\left(\int_{0}^{t} e^{-q s}\|e(s)\|^{q} d s\right)^{1 / q} \cdot
\end{aligned}
$$

Let $\bar{M}=(\|\Omega\|+A H)\left(\Gamma\left(\alpha^{2}\right) / \Gamma^{p}(\alpha) p^{\alpha^{2}}\right)^{1 / p}, \bar{N}=B K\left(\Gamma\left(\alpha^{2}\right) /\right.$ $\left.\Gamma^{p}(\alpha) p^{\alpha^{2}}\right)^{1 / p}$. Then

$$
\begin{aligned}
& \|e(t)\| e^{-t} \\
& \leq\left(\|\phi\| e^{-t}+\bar{N}\|\phi\|\right) \\
& \quad+\left(\bar{M}+\bar{N} e^{-\tau}\right)\left(\int_{0}^{t} e^{-q s}\|e(s)\|^{q} d s\right)^{1 / q} d s .
\end{aligned}
$$

According to Lemmas 7 and 9, one has

$$
\|e(t)\| e^{-t} \leq\left(\|\phi\| e^{-t}+\bar{N}\|\phi\|\right)+\left(\bar{M}+\bar{N} e^{-\tau}\right)
$$

$$
\begin{aligned}
& \cdot\left[\left(1-\left(1-e^{-\left(\bar{M}+\bar{N} e^{-\tau}\right)^{q} t}\right)^{1 / q}\right)^{-q}\right. \\
& \left.\cdot \int_{0}^{t}\left(\|\phi\| e^{-s}+\bar{N}\|\phi\|\right)^{q} e^{-\left(\bar{M}+\bar{N} e^{-\tau}\right)^{q} s} d s\right]^{1 / q} \\
& \leq\left(\|\phi\| e^{-t}+\bar{N}\|\phi\|\right)+\left(\bar{M}+\bar{N} e^{-\tau}\right) q e^{\left(\bar{M}+\bar{N} e^{-\tau}\right)^{q} t} \\
& \cdot\left[\int_{0}^{t}\left(\|\phi\| e^{-s}+\bar{N}\|\phi\|\right)^{q} e^{-\left(\bar{M}+\bar{N} e^{-\tau}\right)^{q} s} d s\right]^{1 / q}
\end{aligned}
$$




$$
\begin{aligned}
& \leq\left(\|\phi\| e^{-t}+\bar{N}\|\phi\|\right)+q(1+\bar{N})\|\phi\| e^{\left(\bar{M}+\bar{N} e^{-\tau}\right)^{q} t} \\
& =\left[e^{-t}+\bar{N}+q(1+\bar{N}) e^{\left(M+N e^{-\tau}\right)^{q} t}\right]\|\phi\| .
\end{aligned}
$$

Therefore

$$
\|e(t)\| \leq\left[1+\bar{N} e^{t}+q(1+\bar{N}) e^{\left[\left(\bar{M}+\bar{N} e^{-\tau}\right)^{q}+1\right] t}\right]\|\phi\| .
$$

Hence, if (26) is satisfied and $\|\phi\|<\delta$, then $\|e(t)\|<\varepsilon$; master system (3) is synchronized with slave system (5) in a finitetime.

Remark 12. References [24-28] discussed the finite-time synchronization of neural networks, which only considered integer-order systems.

Remark 13. Generally, time-delayed differential models unavoidably exist in neural networks [37]. However, quite a few researches investigated the finite-time synchronization of neural networks with delay. Hence, it is foremost important and necessary to consider finite-time synchronization of fractional-order delayed neural networks.

Remark 14. In this paper, Assumptions 4 and 5 are general, not too strict; many functions can satisfy the assumptions; for example, $[15,17,29]$ considered these assumptions too.

Remark 15. In [33], the authors discussed the finite-time synchronization of fractional-order memristor-based neural networks with time delays by using Laplace transform, generalized Gronwall's inequality and Mittag-Leffler functions, and the results showed Mittag-Leffler functions. In this paper, employing Lemma 4 proposed in [35] and Bernoulli inequality can obtain results that only include exponential functions, given that the form is simpler and the calculation is easier.

Remark 16. In Theorem 10, from (12) and (14) and inequality (16), it is obvious that the convergence time $T$ is proportional to the inverse of the control gain $\eta_{i}$. Therefore, a greater $\eta_{i}$ results in the shorter convergence time $T$, and therefore $\eta_{i}$ should be selected in accordance with the convergence time $T$ to be short.

Remark 17. In [32], the authors discussed finite-time stability of fractional-order neural networks with delay by utilizing Gronwall inequality. Unlike the previous work [32], in this paper, we discussed the finite-time synchronization for a class of fractional-order delayed neural networks by employing Lemma 4 proposed in [35], Bernoulli inequality, and inequality skills; two new delay-dependent sufficient conditions have been established.

Remark 18. Different from integer-order delayed systems, it is difficult to construct Lyapunov functions for fractional-order nonlinear systems with time delay; results have been obtained using different techniques from approaches used in the area of finite synchronization. By employing inequality skills, new

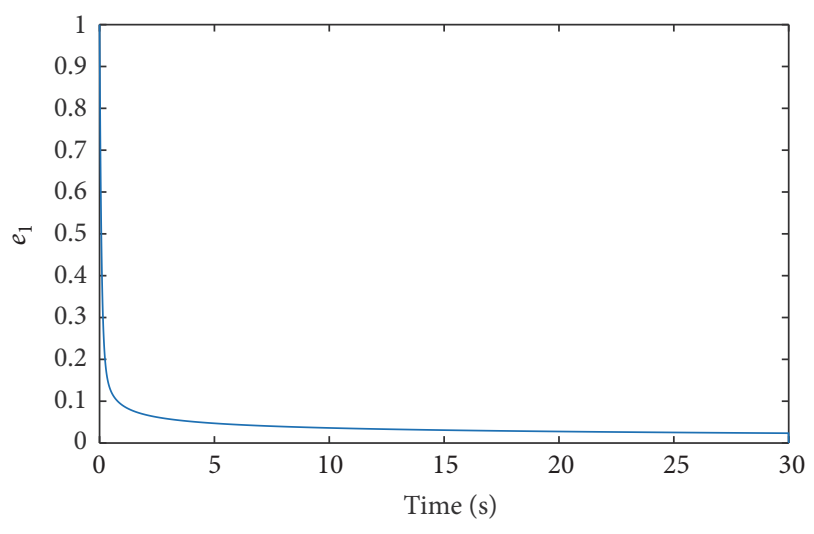

FIGURE 1: The errors state of $e_{1}$ (order $\alpha=0.4$ ).

sufficient conditions ensuring finite-time synchronization are derived.

\section{Numerical Simulations}

Consider the following two-dimensional delayed fractionalorder Hopfield neural networks:

$$
\begin{aligned}
D^{\alpha} x(t)= & -C x(t)+A(t) f(x(t)) \\
& +B(t) g(x(t-\tau))+I
\end{aligned}
$$

for $t \in J=[0,15]$, where $x(t)=\left(x_{1}(t), x_{2}(t)\right)^{T}, \alpha=0.4$ or $\alpha=0.7, \tau=0.1, I=(0,0)^{T}$, and $f_{j}\left(x_{j}(t)\right)=g_{j}\left(x_{j}(t)\right)=$ $\tanh \left(x_{j}(t)\right),(j=1,2)$, denoting $\tanh (x(t))=\left(\tanh \left(x_{1}(t)\right)\right.$, $\left.\tanh \left(x_{2}(t)\right)\right)^{T}$, because of $(\tanh (x))^{\prime}=1-(\tanh (x))^{2} \leq 1$, according to Lagrange Theorem; clearly, $f(x)$ and $g(x)$ satisfy Assumption 4 with $H=K=1, A(t)=\left(\begin{array}{cc}0.2 & -0.1 \\ 0.1 & 0.2\end{array}\right), B(t)=$ $\left(\begin{array}{ll}-0.5 & -0.1 \\ -0.2 & -0.5\end{array}\right), C=\left(\begin{array}{cc}0.1 & 0 \\ 0 & 0.1\end{array}\right)$. So, according to Assumption $5, A=$ $\sup _{t \geq 0}\|A(t)\|=0.3, B=\sup _{t \geq 0}\|B(t)\|=0.7,\|C\|=0.1$. Choose the initial values of master system and slave system as follows: $x_{1}(0)=4, x_{2}(0)=2, y_{1}(0)=3, y_{2}(0)=1$.

When $\alpha=0.4$, take $\delta=0.01, \epsilon=1, \tau=0.1$ and select the control gain $\eta_{1}=4, \eta_{2}=5$; apparently, the condition of Theorem 11 is satisfied. It could be verified that $\bar{M}=8.0815, \bar{N}=1.0674$, and the estimated time of finitetime synchronization is $T=0.7099$. Synchronization errors between master and slave systems are shown in Figures 1 and 2. It is clearly seen that the synchronization errors converge to zero, indicating that master system and slave system are synchronized in a finite-time. For comparison purposes, the curves of the state variable of the master system and the slave system are shown in Figures 3 and 4.

When $\alpha=0.7$, take $\delta=0.01, \epsilon=1, \tau=0.1$ and select the control gain $\eta_{1}=2, \eta_{2}=4$; apparently, the condition of Theorem 10 is satisfied. It is easy to obtain $M=4.2950, N=$ 0.6992 , and the estimated time of finite-time synchronization is $T=0.5937$. Synchronization errors between master and slave systems are shown in Figures 5 and 6 . It can be seen that the synchronization errors converge to zero, confirming that master system and slave system are synchronized in a finitetime. Also, for comparison purposes, the curves of the state 


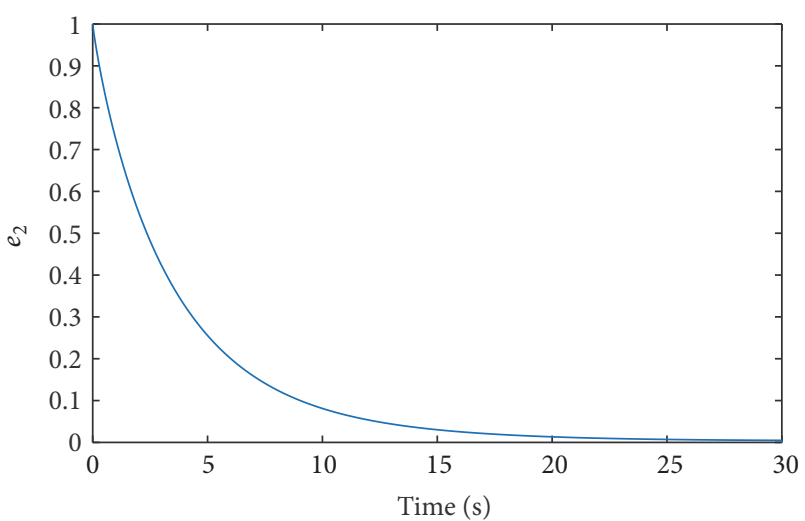

Figure 2: The errors state of $e_{2}(\operatorname{order} \alpha=0.4)$.

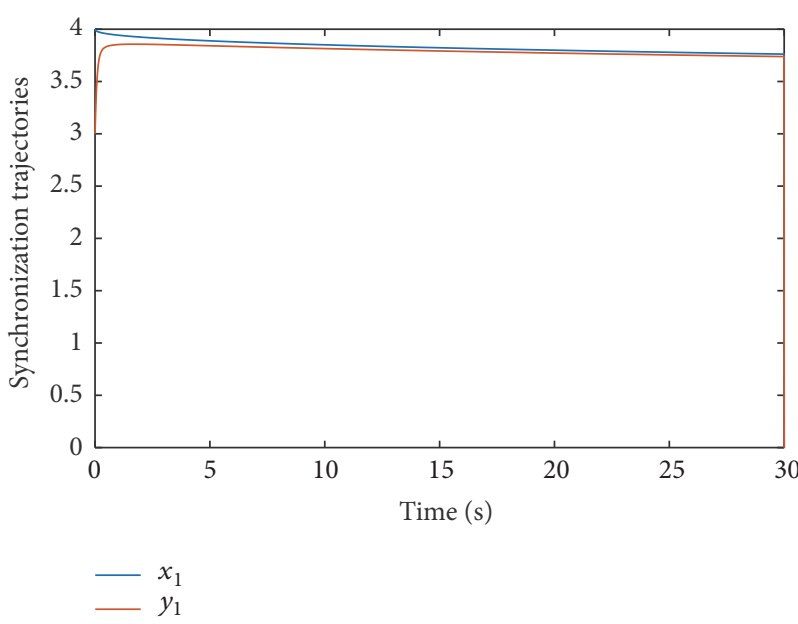

Figure 3: The synchronization trajectories of $x_{1}$ and $y_{1}$ (order $\alpha=$ $0.4)$.

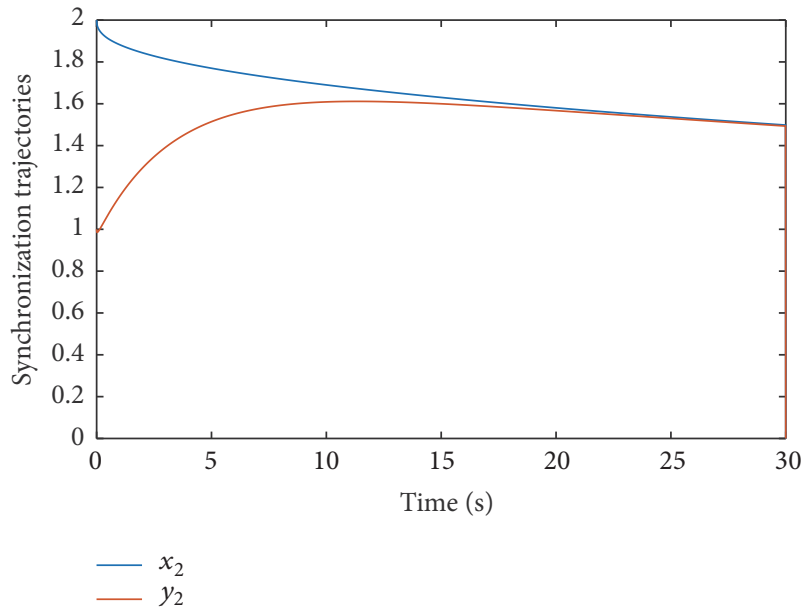

FIGURE 4: The synchronization trajectories of $x_{2}$ and $y_{2}$ (order $\alpha=$ $0.4)$.

variable of the master system and the slave system are shown in Figures 7 and 8.

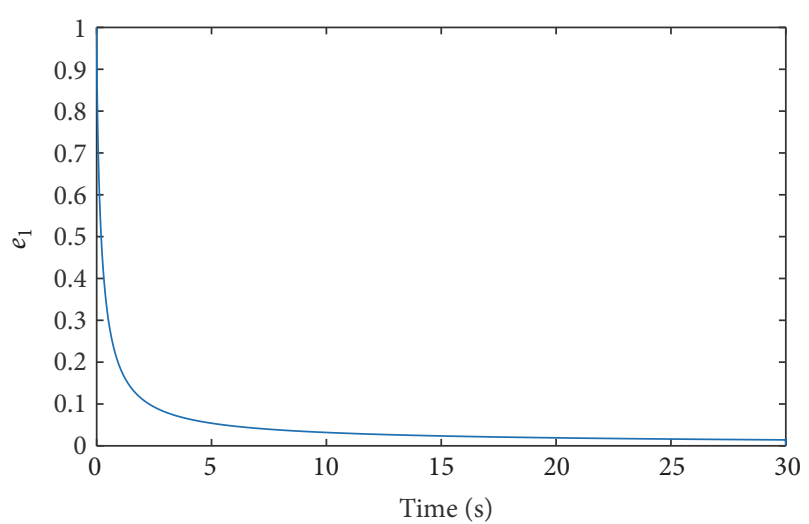

FIGURE 5: The errors state of $e_{1}$ (order $\alpha=0.7$ ).

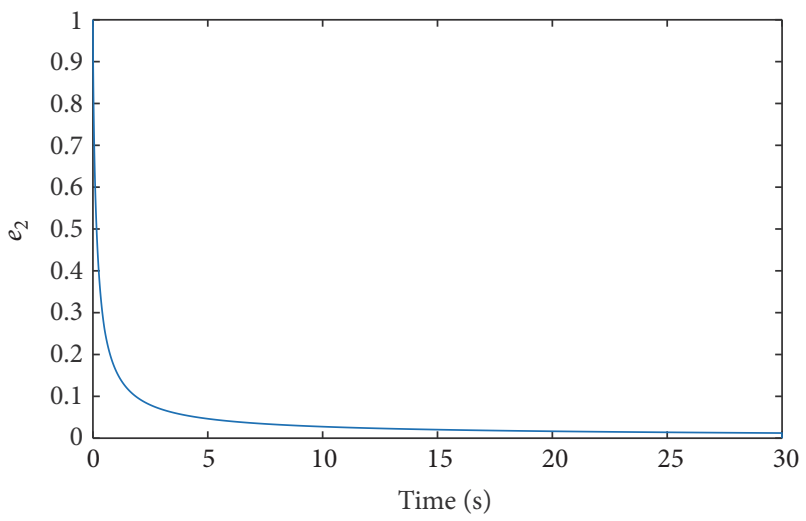

FIGURE 6: The errors state of $e_{2}$ (order $\left.\alpha=0.7\right)$.

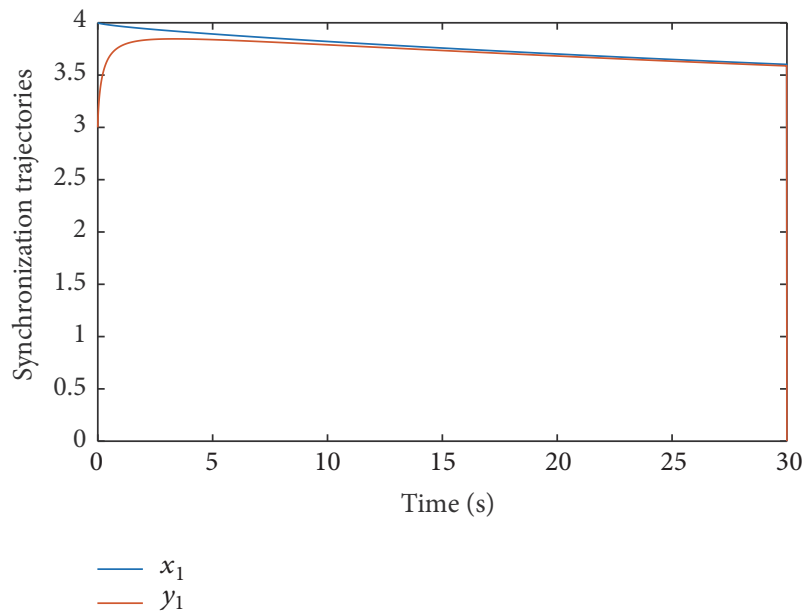

FIgURE 7: The synchronization trajectories of $x_{1}$ and $y_{1}$ (order $\alpha=$ $0.7)$.

\section{Conclusions}

In this paper, finite-time synchronization for a class fractional-order delayed neural networks with order $\alpha$, $0<\alpha \leq 1 / 2$ and $1 / 2<\alpha<1$, was discussed. Some new sufficient conditions are derived to ensure the finite-time synchronization for this class of fractional-order systems. 


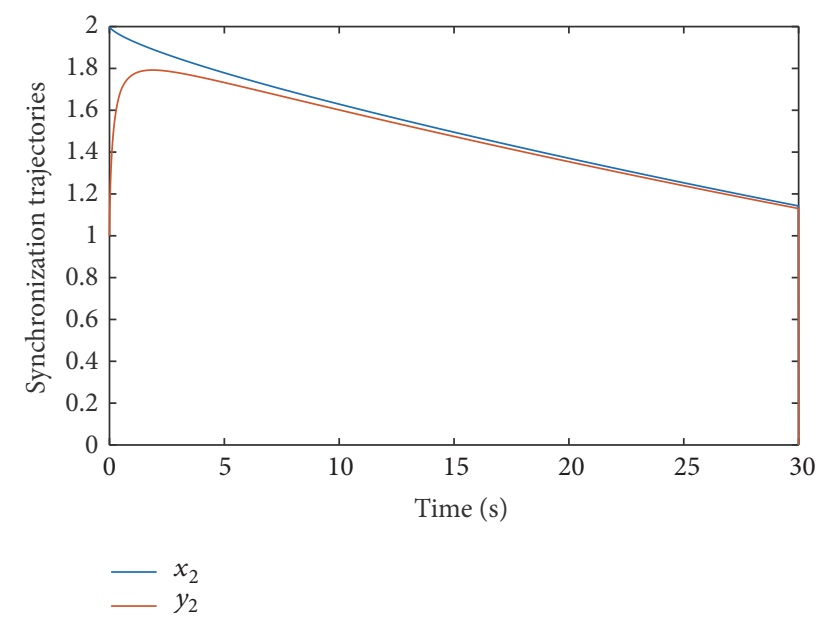

FIGURE 8: The synchronization trajectories of $x_{2}$ and $y_{2}$ (order $\alpha=$ $0.7)$.

Numerical example is presented to verify the effectiveness of the theoretical results. The proposed methods are novel and solve well the finite-time synchronization of fractionalorder delayed neural networks. We would like to point out that it is possible to extend the methods to other fractional-order models, such as fractional-order delayed neutral-type neural networks and fractional neural networks with incommensurate. These issues will be further worth discussing.

\section{Conflicts of Interest}

The authors declare no conflicts of interest.

\section{Acknowledgments}

This work was supported by the National Natural Science Foundation of China (Grant no. 11571016), the Natural Science Foundation of Anhui Province (Grant no. 1608085MA14), and the Natural Science Foundation of the Higher Education Institutions of Anhui Province (Grant no. KJ2015A152).

\section{References}

[1] I. Podlubny, Fractional Differential Equations, Academic Press, New York, NY, USA, 1999.

[2] P. L. Butzer and U. Westphal, "An introduction to fractional calculus," in Applications of Fractional Calculus in Physics, pp. 1-85, World Scientific, Singapore, 2000.

[3] B. B. Mandelbrot, The Fractal Geometry of Nature, Freeman, New York, NY, USA, 1982.

[4] R. Hilfer, Applications of Fractional Calculus in Physics, World Scientific, Singapore, 2000.

[5] A. A. Kilbas, H. M. Srivastava, and J. J. Trujillo, Theory and Applications of Fractional Differential Equations, New York, NY, USA, Elsevier, 2006.
[6] R. Zhang, D. Chen, Y. Do, and X. Ma, "Synchronization and anti-synchronization of fractional dynamical networks," Journal of Vibration and Control, vol. 21, no. 16, pp. 3383-3402, 2015.

[7] H. Liu, S. Li, J. D. Cao, A. G. Alsaedi, and F. E. Alsaadi, "Adaptive fuzzy prescribed performance controller design for a class of uncertain fractional-order nonlinear systems with external disturbances," Neurocomputing, vol. 219, pp. 422-430, 2017.

[8] B. Xu, D. Chen, H. Zhang, and R. Zhou, "Dynamic analysis and modeling of a novel fractional-order hydro-turbine-generator unit," Nonlinear Dynamics, vol. 81, no. 3, pp. 1263-1274, 2015.

[9] B. B. Xu, D. Y. Chen, H. Zhang, and F. Wang, "The modeling of the fractional-order shafting system for a water jet mixedflow pump during the startup process," Communications in Nonlinear Science and Numerical Simulation, vol. 29, no. 1-3, pp. 12-24, 2015.

[10] B. Xu, F. Wang, D. Chen, and H. Zhang, "Hamiltonian modeling of multi-hydro-turbine governing systems with sharing common penstock and dynamic analyses under shock load," Energy Conversion and Management, vol. 108, pp. 478-487, 2016.

[11] B. Xu, D. Chen, H. Zhang, F. Wang, X. Zhang, and Y. Wu, "Hamiltonian model and dynamic analyses for a hydro-turbine governing system with fractional item and time-lag," Communications in Nonlinear Science and Numerical Simulation, vol. 47, pp. 35-47, 2017.

[12] L. Chen, J. Qu, Y. Chai, R. Wu, and G. Qi, "Synchronization of a class of fractional-order chaotic neural networks," Entropy. An International and Interdisciplinary Journal of Entropy and Information Studies, vol. 15, no. 8, pp. 3265-3276, 2013.

[13] S. Zhang, Y. Yu, and W. Hu, "Robust stability analysis of fractional-order Hopfield neural networks with parameter uncertainties," Mathematical Problems in Engineering, vol. 2014, Article ID 302702, 14 pages, 2014.

[14] J. Chen, Z. Zeng, and P. Jiang, "Global Mittag-Leffler stability and synchronization of memristor-based fractional-order neural networks," Neural Networks, vol. 51, pp. 1-8, 2014.

[15] H.-B. Bao and J.-D. Cao, "Projective synchronization of fractional-order memristor-based neural networks," Neural Networks, vol. 63, pp. 1-9, 2015.

[16] D. Chen, R. Zhang, X. Liu, and X. Ma, "Fractional order Lyapunov stability theorem and its applications in synchronization of complex dynamical networks," Communications in Nonlinear Science and Numerical Simulation, vol. 19, no. 12, pp. 4105-4121, 2014.

[17] H. Wu, L. Wang, Y. Wang, P. Niu, and B. Fang, "Global mittagleffler projective synchronization for fractional-order neural networks: an lmi-based approach," Advances in Difference Equations, vol. 132, pp. 1-18, 2016.

[18] R. Rakkiyappan, R. Sivasamy, and X. Li, "Synchronization of Identical and Nonidentical Memristor-based Chaotic Systems Via Active Backstepping Control Technique," Circuits, Systems, and Signal Processing, vol. 34, no. 3, pp. 763-778, 2015.

[19] D. Chen, R. Zhang, X. Ma, and S. Liu, "Chaotic synchronization and anti-synchronization for a novel class of multiple chaotic systems via a sliding mode control scheme," Nonlinear Dynamics. An International Journal of Nonlinear Dynamics and Chaos in Engineering Systems, vol. 69, no. 1-2, pp. 35-55, 2012.

[20] N. Li and J. Cao, "New synchronization criteria for memristorbased networks: Adaptive control and feedback control schemes," Neural Networks, vol. 61, pp. 1-9, 2015.

[21] E. Ahmed and A. S. Elgazzar, "On fractional order differential equations model for nonlocal epidemics," Physica A: Statistical Mechanics and Its Applications, vol. 379, no. 2, pp. 607-614, 2007. 
[22] H. Liu, S. Li, H. Wang, Y. Huo, and J. Luo, "Adaptive synchronization for a class of uncertain fractional-order neural networks," Entropy. An International and Interdisciplinary Journal of Entropy and Information Studies, vol. 17, no. 10, pp. 7185-7200, 2015.

[23] Y. Pan, Y. Liu, B. Xu, and H. Yu, "Hybrid feedback feedforward: An efficient design of adaptive neural network control," Neural Networks, vol. 76, pp. 122-134, 2016.

[24] A. Abdurahman, H. Jiang, and Z. Teng, "Finite-time synchronization for memristor-based neural networks with timevarying delays," Neural Networks, vol. 69, pp. 20-28, 2015.

[25] C. Hu, J. Yu, and H. Jiang, "Finite-time synchronization of delayed neural networks with Cohen-Grossberg type based on delayed feedback control," Neurocomputing, vol. 143, pp. 90-96, 2014.

[26] J. Huang, C. Li, T. Huang, and X. He, "Finite-time lag synchronization of delayed neural networks," Neurocomputing, vol. 139, pp. 145-149, 2014.

[27] W. Xiong and J. Huang, "Finite-time control and synchronization for memristor-based chaotic system via impulsive adaptive strategy," Advances in Difference Equations, 2016:101, 9 pages, 2016.

[28] W. Cui, J.-A. Fang, and S. Su, "Finite time synchronization problems of delayed complex networks with stochastic perturbations," Advances in Difference Equations, vol. 149, pp. 1-9, 2014.

[29] X. Li and S. Song, "Stabilization of Delay Systems: delaydependent Impulsive Control," IEEE Transactions on Automatic Control, vol. 62, no. 1, pp. 406-411, 2017.

[30] X. Li, R. Rakkiyappan, and N. Sakthivel, "Non-fragile synchronization control for Markovian jumping complex dynamical networks with probabilistic time-varying coupling delays," Asian Journal of Control, vol. 17, no. 5, pp. 1678-1695, 2015.

[31] X. Li, X. Zhang, and S. Song, "Effect of delayed impulses on input-to-state stability of nonlinear systems," Automatica. A Journal of IFAC, the International Federation of Automatic Control, vol. 76, pp. 378-382, 2017.

[32] R. Wu, Y. Lu, and L. Chen, "Finite-time stability of fractional delayed neural networks," Neurocomputing, vol. 149, pp. 700707, 2015.

[33] G. Velmurugan, R. Rakkiyappan, and J. Cao, "Finite-time synchronization of fractional-order memristor-based neural networks with time delays," Neural Networks, vol. 73, pp. 36-46, 2016.

[34] D. S. Mitrinović and P. M. Vasić, Analytic Inequalities, Springer, Berlin, Germany, 1970.

[35] C. Li and W. Deng, "Remarks on fractional derivatives," Applied Mathematics and Computation, vol. 187, no. 2, pp. 777-784, 2007.

[36] D. Willett, "Nonlinear vector integral equations as contraction mappings," Archive for Rational Mechanics and Analysis, vol. 15, pp. 79-86, 1964.

[37] X. Li and S. Song, "Impulsive control for existence, uniqueness, and global stability of periodic solutions of recurrent neural networks with discrete and continuously distributed delays," IEEE Transactions on Neural Networks and Learning Systems, vol. 24, no. 6, pp. 868-877, 2013. 


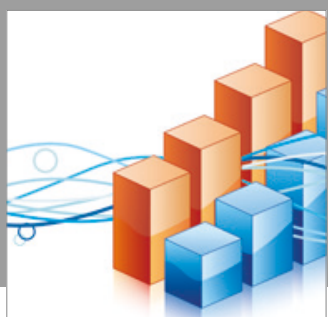

Advances in

Operations Research

vatersals

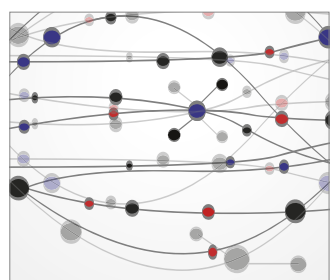

\section{The Scientific} World Journal
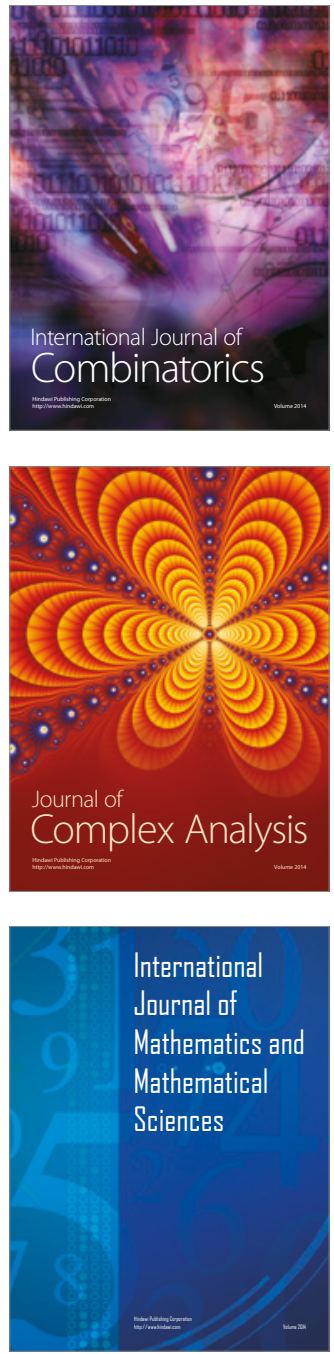
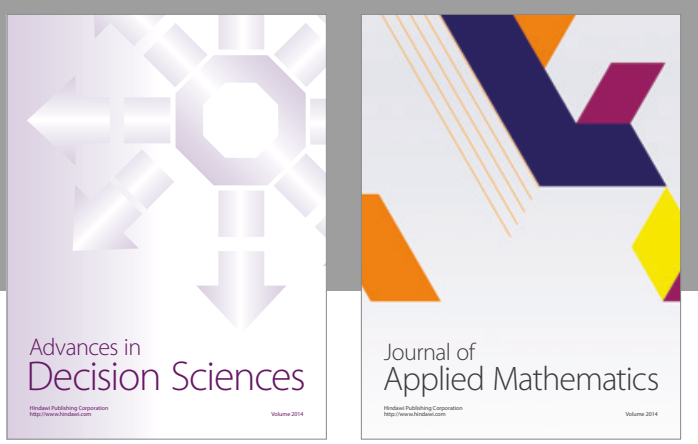

Algebra

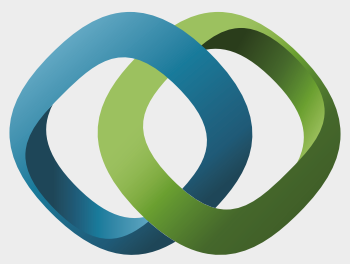

\section{Hindawi}

Submit your manuscripts at

https://www.hindawi.com
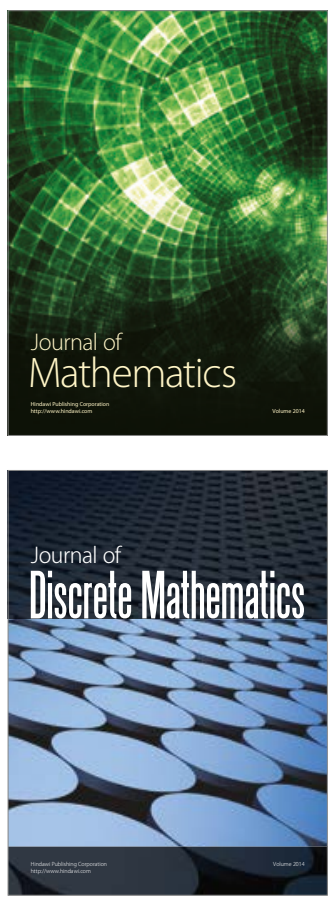

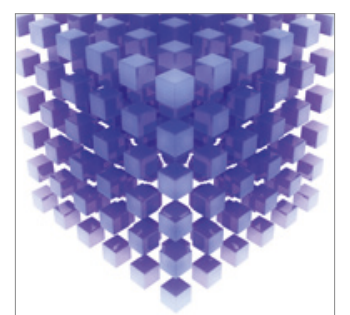

Mathematical Problems in Engineering
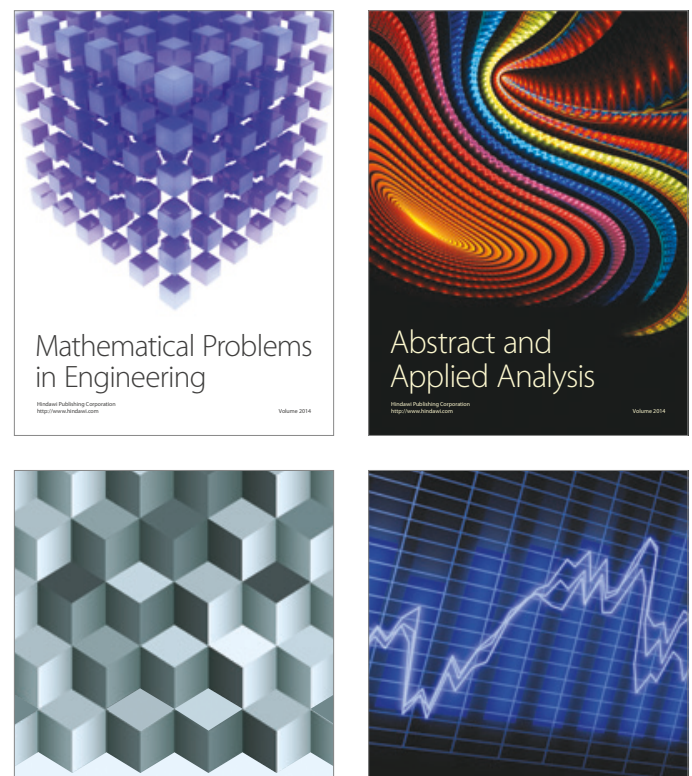

Journal of

Function Spaces

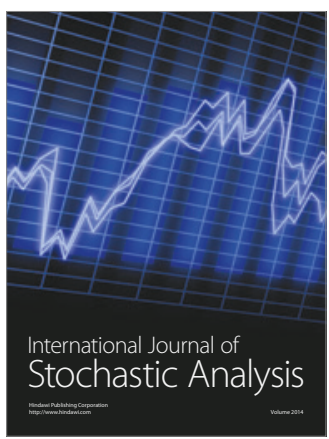

Probability and Statistics
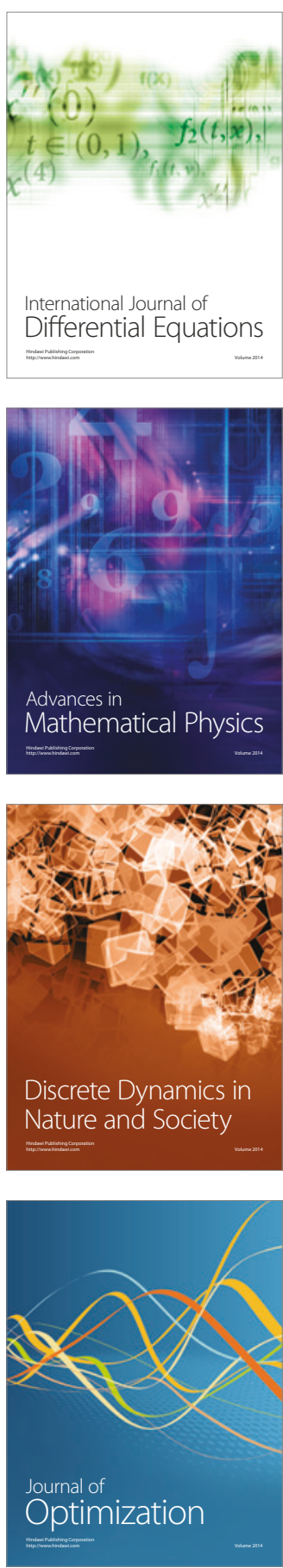